are not tolerated. Unfortunately, several of those perceived errors are the result of no more than our natural inability to predict the future accurately all the time, compounded in the case of psychiatrists by the expectation that we will somehow eradicate any violence to the public from anyone who has ever consulted a psychiatrist - and this in spite of any adequate legislation. This is supported by the evidence that over $85 \%$ of suspended consultants are reinstated because the complaints against them have been found to be unjust, but these unjustly suspended doctors suffer stress and humiliation and their colleagues become increasingly fearful that the same may happen to them. Genuine errors of judgment will also occasionally occur because doctors are only human like everyone else.

It is therefore my opinion that for the Colleges to believe that their "limitations as self-proclaimed guardians of high clinical standards" have been exposed is to play into this damaging prevailing philosophy. To expect that audits and guidelines, national or otherwise, will eradicate all errors is a very dangerous assumption because it is patently false.

Attempting to practise in a culture where human error is not tolerated and doctors daily fear accusation has taken its toll and has resulted in a drop in recruitment and a rush of early retirements, certainly where psychiatry is concerned. If the Colleges feed into this culture by forging "an alliance either with NHS employers or with the General Medical Council in order to obtain the powers over qualified specialists which they currently lack", this must be done very sensitively or their members are likely to feel even more criticised and unsupported and morale will surely plummet further and this is not ultimately in the public interest.

The Royal College of Psychiatrists has launched what would seem to be an excellent public campaign to reduce stigma against people suffering from mental health problems. This is laudable, but it is a common perception of its members that they too are being increasingly stigmatised and vilified for reasons which are outside of their control. It is important for the College to appreciate this and to consider addressing this issue, perhaps by another public campaign tackling public attitudes, awareness and understanding of what the nation can reasonably expect of its psychiatrists. There need not necessarily be a conflict in furthering the interests of both patients and their doctors, and indeed as members pay their Colleges sizeable subscriptions most will expect that their Colleges will look after their interests in return.

ANNe Cremona, Consultant Psychiatrist, 2 Maids of Honour Row, Richmond-upon-Thames, Surrey TW9 1NY

\section{Psychiatry and the Mental Health Act}

Sir: We would like to thank Szmukler \& Holloway (Psychiatric Bulletin. December 1998, 22 662-665) for their comments on the Mental Health Act. Their review article raises important questions. However, while we agree with many of their ideas, we believe that there are substantial problems with their position. They argue that current mental health legislation is both contradictory and discriminatory, as recently highlighted by the $L$. $v$. Bournewood 1997 Court of Appeal judgement. They propose that Mental Health Act legislation should be replaced by an 'Incapacity Act', which would apply to mental and physical illness, and 'dangerousness' legislation to cover the need for public protection. These proposals contain much that is to be commended. A mental capacity act with statutory rights to advance directives, patient advocacy and judicial appeals against treatment is infinitely better than more coercive legislation.

However, we agree with Fulford's (1998) argument that equating bodily and mental illness is simplistic. This is implied both in the paper by Szmuckler \& Holloway and the linked editorial by Zigmond (1998). For example, Szmukler \& Holloway declare that: "there is no logical reason to discriminate between mental incapacity occasioned by mental disorder and physical disorder" and Zigmond says: "this (Medical Incapacity Act) would provide for the medical treatment, both mental and physical, of those who lack capacity from whatever cause". Our clinical experience, rooted in our daily contact with people in severe distress, has led us substantially to question the accepted wisdom that distress can be adequately grasped within a medical idiom based on concepts such as pathology, diagnosis, investigation, treatment and prognosis. Human experience resists reduction to causal scientific models. Most of our work, as psychiatrists, is concerned with the interpretation of behaviour in relation to individual personal and social contexts, not the explanation of this behaviour in linear causal terms. We believe that while the medical sciences, upon which psychiatry is based, can inform our interpretations of madness and distress, they can do so in a limited way only. Historically, this explanatory potential of psychiatry has been exaggerated and thus its power to predict behaviour. As a result, society has invested it with the power to detain and to treat patients against their will. We believe that it is time for psychiatry to give up both this power and the associated idea that it can render mental disorder within a scientific paradigm. The two issues are inextricably linked. If we continue to maintain the latter, the implication is that medical perspectives on mental 
incapacity will continue to attract a legitimacy which far outweighs their validity. The power of psychiatry will continue as before.

We also disagree with Zigmond's (1998) idea that a Medical Incapacity Act would reduce stigma at a stroke, by offering the same protection to all patients unable to consent to medical interventions. Stigma is primarily a cultural, not a legal, issue. Media representations of mental disorder and distress are currently the greatest problem. Journalists have managed to connect the issues of dangerousness and mental illness in the imagination of both public and politicians and, as a result, tolerance towards people with mental health problems is at an all time low. This can only be combatted by a joint campaign of users and professionals. In turn, this will only happen if professionals begin to question the politics of mental health and the limitations of their knowledge in an open way. If psychiatrists continue to assert a simple equation between bodily and mental illness they will miss an historic opportunity to open up a new agenda in the area of mental health.

FULFORD, K. M. (1998) Invited commentaries on: Mental health legislation is now a harmful anachronism. Psychiatric Bulletin, 22, 666-668.

Zigmond, A. S. (1998) Medical Incapacity Act. Psychiatric Bulletin. 22, 657-658.

P. BRACKEN, Consultant Psychiatrist and PHILIP THOMAS, Bradford Home Treatment Service, Edmund Street Clinic, 26 Edmund Street, Bradford BD5 OBJ

\section{Mental incapacity}

Sir: Szmukler \& Holloway (Psychiatric Bulletin, December 1998; 22, 662-665) are misguided to suggest that incapacitated patients would be afforded better protection by the adoption of an Incapacity Act along the lines proposed by the Government Green Paper Who Decides (Lord Chancellor's Department, 1997).

The Green Paper gives no guidance as to how, or by whom, incapacity is to be judged and takes no account of 'shades' of capacity or temporary incapacity. Once patients are designated as 'incapacitated', a previously drafted 'living will' may come into force which requires 'treatment', possibly including food and fluids, to be withdrawn, leading to death by dehydration or starvation. Gardner et al (1985) showed that patients change their minds when illness strikes them; however, it would be hard for patients to change or withdraw advance directives if they had already been classified as incapacitated. Moreover, suicide notes may under this legislation constitute advance statements. Cries for help could become death warrants. The Bill also makes legal non-consensual medical procedures (Clause 10) and research (Clause 11) on incapacitated' patients, even if of no benefit to them. This could include organ removal from a non-dying patient.

Doctors attempting to resist any of the possibilities discussed could be liable to criminal prosecution. Rather than providing the extra protection to mentally incapacitated people which Szmukler \& Holloway so laudably seek, this Bill would make possible widespread abuse of these patients and lead to a fatal compromise in medical ethics. Our profession should therefore resist it at all costs.

GaRDNER. B. P.. THEOCLEOUS, F., WATT, J. W. H. et al (1985) Ventilation or dignifled death for patients with high tetraplegia. British Medical Journal, 291, 1620-1622.

LORD ChanCELLOR'S DePARTMENT (1997) Who Decides? Making Decisions on Behalf of Mentally Incapacitated Adults. London: HMSO.

DAVID KINGSLEY, Basic Specialist Trainee in Old Age Psychiatry. West Lancashire NHS Trust. Ormskirk, Lancashire L39 2AZ

\section{Participation in continuing professional development}

Sir: Contrary to Weaver's assertion (Psychiatric Bulletin, December 1998, 22, 771), my editorial (Psychiatric Bulletin, September 1998, 22, 529530) did not speculate in any way on the continuing professional development (CPD) activities of psychiatrists who have not registered with our College-based scheme. I regret that Weaver seems to have missed the crucial point of my article, which set out to emphasise how important it is that our CPD scheme should be given all possible support. Participation in CPD is only part of what is expected of us. We also need to demonstrate clearly that we have done so. Our scheme is surely the best way to coordinate this process and ultimately demonstrate its effectiveness.

As I predicted, things have now moved apace and some form of revalidation is a near certainty. I can only guess at what this will entail, but I do believe that a well supported College-based scheme should offer psychiatrists several distinct benefits. It is conceivable that Collegebased CPD credentials might be taken into account in the revalidation process, and they should help to maintain a standard of excellence which is set nationally. A recent survey of attitudes to our scheme has shown that among a sample of consultants who have not registered for CPD, the most common reason for not doing so is an excessive clinical work load. Here too a College based scheme should be well placed to challenge relevant employing NHS trusts in a 